\title{
Utility of Video Electroencephalography Monitoring for Diagnosis of Epilepsy and Nonepileptic Paroxysmal Events
}

\author{
Diana Diaz-Cortés ${ }^{1 *}$, Lorena Vega-Zelaya ${ }^{2,3 *}$, Laura López ${ }^{2}$, María Toledo ${ }^{4}$ and Jesús Pastor ${ }^{2,3}$ \\ ${ }^{1}$ Neurology, Clínica Palermo Bogotá, Colombia \\ ${ }^{2}$ Clinical Neurophysiology Hospital Universitario La Princesa Madrid, Spain \\ ${ }^{3}$ Fundación de Investigación La Princesa, Hospital Universitario La Princesa Madrid, Spain \\ ${ }^{4}$ Neurology, Hospital Universitario de La Princesa \\ *Both Authors Contributed Equally
}

*Corresponding authors: Dr. Jesús Pastor, Neurofisiología Clínica, Hospital Universitario de La Princesa, C/Diego de León 62, Madrid 28006, SPAIN; Tel: 34-91-5202213; Fax: 34-91-4013582; E-mail: jesus.pastor@salud.madrid.org

Dr. Diana Diaz-Cortés, Neurology, Clínica Palermo Bogotá, Colombia.

Received: May 19, 2020; Accepted: May 25, 2020; Published: May 27, 2020

\begin{abstract}
Objective: Video electroencephalography monitoring (VEM) is an important tool for the diagnosis and classification of seizures and for the presurgical evaluation of patients with drug-resistant epilepsy. The aim of this study was to assess the utility of VEM in patients referred for differential diagnoses (epileptic events versus nonepileptic episodes) and/or improving diagnosis accuracy in epilepsy.

Methods: Three hundred and eight VEM studies were analyzed retrospectively over a period of 3 years. Only studies obtaining seizure classification and diagnostic clarification to determine the nature of paroxysmal events were included $(n=125)$. The clinical diagnoses before and after VEM were compared. VEM was useful if it changed the diagnosis and/or therapy or if it answered the clinical question raised by the referring physician.

Results: One hundred twenty-five patients were included (64\% women) with a mean age of $43.0 \pm 1.6$. During VEM, 61 patients had typical clinical events; there were 21 seizures, 25 physiological events, and 18 psychogenic nonepileptic seizures (PNES). In the PNES group, we found that women and younger patients were more frequent. Epileptic patients had a shorter evolution time, and the physiological events group had an older age at event onset compared to the epilepsy group. The provisional diagnosis changed in 35\% of the cases after VEM. The diagnostic usefulness of VEM was $89 \%$. After VEM, treatment changed in $50 \%$ of patients.
\end{abstract}

Significance: VEM is an essential tool to differentiate seizure from nonepileptic paroxysmal events. It is imperative to achieve an accurate diagnosis to determine the most suitable therapeutic approach.

Keywords: epileptic seizures, nonepileptic paroxysmal events, physiological events, psychogenic nonepileptic seizure, syncope

\section{Key Point Box}

- Misdiagnosis of nonepileptic paroxysmal events and epilepsy represents a problem with important therapeutic and social repercussions.

- Inpatient VEM has been demonstrated to be a useful tool for the diagnosis and classification of seizure events.

- VEM achieved an accurate diagnosis and accomplished the most suitable therapeutic approach in epileptic and nonepileptic patients.

\section{Introduction}

An Epileptic Seizure (ES) is defined as a transient occurrence of behavioral alterations produced by abnormal, excessive, and hypersynchronous neuronal activity in the brain $[1,2]$. However, since the symptoms are diverse, diagnosis of ES may be challenging given the differential diagnoses $[3,4]$. Nonepileptic paroxysmal events of physiological and psychological origin, such as syncope, sleep disorders, migraines or psychogenic nonepileptic seizures (PNES), can also manifest as behavioral disturbance events or transient alterations of consciousness [3]. It is extremely important to achieve an accurate diagnosis in epilepsy, given its morbidity associated with undiagnosed and untreated seizures [2]. In the same way, misdiagnosed epilepsy can result in side effects from antiepileptic drugs, economic costs, and impact on quality of life.

Video electroencephalography monitoring (VEM) is the most useful diagnostic tool for the classification of ES, as well as being the current gold standard for distinguishing epileptic versus nonepileptic paroxysmal events [5]. The International League Against Epilepsy 
(ILAE) recommends VEM for i) differential diagnosis for epileptic seizures, ii) characterization and classification of seizures types and epilepsy syndrome, iii) quantifying seizures, iv) intensive care unit monitoring, and v) presurgical evaluation of drug-resistant epilepsy [6].

Long-term VEM (1-6 days) has been shown to improve diagnostic accuracy compared with standard EEG (20-30 minutes) [7]. VEM not only obtains more complete information regarding the EEG background and characterization of the interictal activity but also analyzes the clinical semiology with the electrophysiological phenomenology during clinical events [8]. Moreover, during preoperative evaluation of drug-resistant temporal lobe epilepsy patients, it has been shown that no other routine tests, including imaging studies, were as reliable as VEM in identifying and characterizing epilepsy seizures and defining the epileptogenic zone in patients evaluated for epilepsy surgery [9]. Nonetheless, VEM is an expensive tool that needs sophisticated equipment, highly qualified staff, and admission of patients to the hospital during variable periods.

A highly variable range of diagnostic usefulness for VEM has been described (19\%-75\%), which depends first on how utility is defined and on the selection of the patients evaluated [10]. However, another factor is also involved in this issue. There is no standard protocol for the duration of VEM. In fact, some units carry out 12-hour studies, while in other units, monitoring lasts several days.

In our unit, we systematically used two protocols of VEM: 24hour VEM for the differential diagnosis and follow-up of epilepsy patients and a longer-lasting VEM (2-10 days) for the presurgical evaluation of drug-resistant epilepsy.

The purpose of this study was to assess the diagnostic utility of VEM for the classification and differential diagnosis of epilepsy and nonepileptic paroxysmal events in a national reference unit for refractory epilepsy. We define a VEM study as useful when either a tentative diagnosis was changed or confirmed or when patient management was modified as a result of the information obtained from VEM.

\section{Methods}

\section{Patients}

We retrospectively analyzed the clinical chart and VEM records of consecutive patients who underwent inpatient VEM at the video electroencephalography (VEEG) unit of the National Reference Unit for Refractory Epilepsy at Hospital Universitario de la Princesa, over a period of three years $(n=308)$. Only those patients who had been referred to i) differentiate between epileptic and nonepileptic events and/or ii) to classify the kind of seizure and epilepsy syndrome type were selected. Finally, the number of patients fulfilling these conditions was 125 . Those patients with known medically refractory epilepsy undergoing presurgical evaluation were excluded.

Clinical charts, including age, sex, age at symptomatology onset, duration, provisional clinical diagnosis, antiepileptic drugs (AED), brain magnetic resonance imaging (MRI), and ambulatory standard EEG, were revised. In cases where patients had undergone an ambulatory EEG and neuroimaging at institutions other than our hospital, only the reports were available, and the studies themselves were not reviewed.

The provisional diagnosis of the physician who referred the patient to the VEEG unit (pre-VEM diagnosis) was compared with the final clinical diagnostic (post-VEM diagnosis), and both diagnoses were classified into the following categories: i) epilepsy, ii) PNES, and iii) nonepileptic paroxysmal events of physiological origin, including a cardiogenic or metabolic cause or event-related to other neurological diseases (e.g., sleep disorders, movement disorders, migraine, or cognitive disturbance).

\section{Video electroencephalography monitoring}

VEM was performed using a 64-channel digital VEEG system (EMU64, NeuroWorks. XLTEK ${ }^{\oplus}$, Oakville, Canada) with 19 scalp stainless steel electrodes fixed with collodion according to the 10-20 international system; electrocardiography (ECG) and simultaneous video images were recorded continuously for $24 \mathrm{~h}$. If needed, one or two electromyography channels (EMG) were added, too. Recordings were performed at a $512 \mathrm{~Hz}$ sampling rate with a $0.5-70 \mathrm{~Hz}$ bandwidth, $50 \mathrm{~Hz}$ notch on. EMG bandwidth was $1.5-200 \mathrm{~Hz}$, notch on, and ECG bandwidth was $1.5-30 \mathrm{~Hz}$, notch on. Impedances for EEG were under $25 \mathrm{k} \Omega$.

Patients had partial sleep deprivation, but medication withdrawal was not undertaken. If considered, induction techniques involving suggestion and administration of placebo were used in some patients [4].

To avoid biases in the assessment of VEM utility, we considered three periods chronologically: i) $t_{1}$ defining the putative diagnosis and treatment considered as the basal line usually performed 1-2 months before, ii) VEM (performed at $t_{V E M}$ ), and finally, iii) the period $t_{2}$ that includes the first clinical interview after VEM (usually 1-2 months later). No other complementary studies were undertaken between $t_{1}$ and $t_{2}$; thus, we were sure that changes either in diagnosis or treatment would be due to the VEM result.

The patient's function state $(P)$ is defined at time $t$, as a twovariables function, i.e., treatment $(T)$ and diagnosis $(d)$, stated as $p_{t}(T, d)$ . In this definition, $t$ is not a variable but a parameter. Therefore, we have different possibilities of changes at consecutive periods (Figure 1), depending on either $T$ or $d$ or both changing between $t_{1}$ and $t_{2}$. VEM was considered useful when variables $T, d$ or both changed.

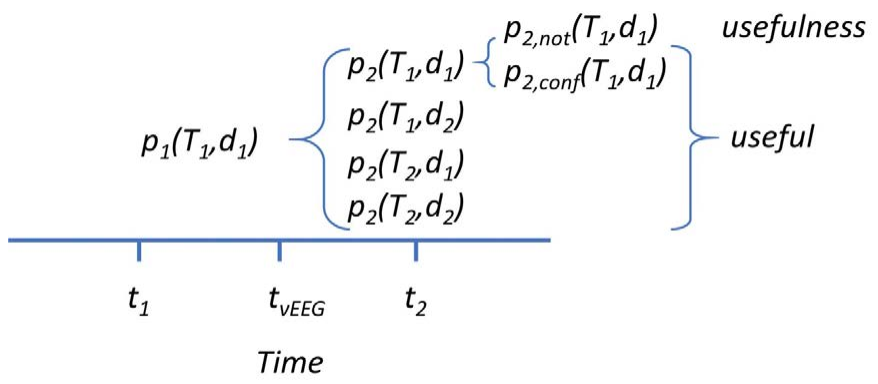

Figure 1: Scheme used to evaluate the utility of VEM. 
However, in some cases where both $T$ and $d$ remained the same, the study can still be considered useful if it confirms a previously suspected but not well-established diagnosis, e.g., suspected PNES with no pharmacological treatment where, after VEM, a positive result confirms PNES. In such cases, the utility derived from confirmation is written as $p_{1}\left(T_{1}, d_{1}\right) \rightarrow p_{2, \text { conf }}\left(T_{1}, d_{1}\right)$. In other words, only when the VEM result did not change any variable or confirm a suspected previous diagnosis $\left(p_{2, \text { not }}\left(T_{1}, d_{1}\right)\right)$ was it considered to be not useful.

Additional, clinical demographics and VEM bioelectrical features for each of the three clinical diagnostic categories were also analyzed.

\section{Statistical analysis}

Statistical comparisons between groups were performed using Student's $t$-test or ANOVA for data with normal distribution. Normality was evaluated using the Kolmogorov-Smirnov test. The Mann-Whitney rank sum test or ANOVA on ranks was used when normality failed. In the last case, Dunn's method was used for all pairwise post hoc comparisons of mean ranks of treatment groups. Chi-square test $\left(x^{2}\right)$ was used to assess the differences between groups of patients.

The SigmaStat 3.5 software (SigmaStat, Point Richmond, CA, USA) was used for statistical analysis. The significance level was set at $\mathrm{p}<0.05$. The results are presented as the mean \pm SEM, except where otherwise indicated.

\section{Results}

\section{Patients}

A total of 125 patients were included ( $64 \%$ women) with a mean age of $43.0 \pm 1.6$ years. The mean disease duration was $8.8 \pm 1.0$ years. A total of $82(66 \%)$ patients received treatment with AED at the time of the study, and the mean number of AED was $1.3 \pm 0.1$. Brain magnetic resonance imaging (MRI) was available in 113 patients, with abnormal findings in 57 (50\%) patients; of these, only 8 patients (14\%) had epileptogenic lesions. Standard EEG was performed in 92 patients, $37 \%$ of which displayed epileptiform activity, $24 \%$ was reported as abnormal nonepileptic activity, and 39\% was normal.

Regarding the provisional clinical diagnosis, ES was suspected in 67 patients (54\%). Nonepileptic events of physiological origin were suspected in 45 patients, accounting for syncope (30 patients), movement disorders ( 7 patients), cognitive impairment (3 patients), sleep disorders (1 patient), and others (4 patient). A diagnosis of PNES was suspected in 10 patients, and in three of them, it was considered that it could also coincide with the diagnosis of epilepsy.

\section{Video electroencephalography monitoring}

During VEM, typical events, described as similar to accustomed, occurred in 61 patients (49\%). Of these, 21 patients (36\%) had ES, 25 patients (41\%) had nonepileptic paroxysmal events of physiological origin and in 13 cases (21\%), the diagnosis was PNES. One of these latter patients had ES along with PNES.

Regarding patients who did not have typical events ( $\mathrm{n}=$ 65 ), we found epileptiform activity in 36 patients (37.5\%) and nonepileptiform abnormalities in 20 patients (21.5\%). Table 1 shows the electroencephalographic findings in all patients.

The three diagnostic categories, before and after VEM, are shown in Table 2. Patients with the double diagnosis of epilepsy and PNES have been included. As we can observe from this table, the diagnosis of epilepsy was confirmed in $41 \%$ of patients, $24 \%$ less than the initial presumptive diagnosis. Additionally, the diagnosis of PNES increased by $80 \%$ after VEM. In five patients, diagnosis of both epilepsy and PNES was made.

We compared the demographic characteristics between patients with final clinical diagnosis of epilepsy, events of physiological origin, and PNES (Table 3). We found that in the PNES group, women were more frequent in comparison with the other two groups. Additionally, this group had the lowest average age compared to nonepileptic events of physiological origin group. This last group had the highest mean age of onset of symptoms compared to the other two groups. It also had the shorter evolution time compared with the epilepsy group.

On the other hand, epileptiform activity on standard EEG was associated with the occurrence of epilepsy diagnosis compared with physiological events and PNES. Moreover, patients with epilepsy (59.6\%) were more likely to have an abnormal brain MRI scan.

We assessed the overall structure for all the three groups according

Table 1. VEM findings in patients with and without typical events. Between brackets is shown percentage

\begin{tabular}{|l|c|c|c|}
\hline Electroencephalographic diagnosis & $\begin{array}{c}\text { ES } \\
(\mathbf{N}=\mathbf{2 2})^{*}\end{array}$ & $\begin{array}{c}\text { NEE } \\
(\mathbf{N}=\mathbf{3 9})^{*}\end{array}$ & $\begin{array}{c}\text { No events } \\
(\mathbf{N}=\mathbf{6 5})\end{array}$ \\
\hline Epileptiform activity & $21(95 \%)$ & $15(38 \%)$ & $36(55 \%)$ \\
\hline Non-epileptiform abnormalities & $1(5 \%)$ & $9(23 \%)$ & $10(15 \%)$ \\
\hline Encephalopathy & - & $1(3 \%)$ & $3(4 \%)$ \\
\hline Normal & - & $14(36 \%)$ & $17(26 \%)$ \\
\hline
\end{tabular}

"One patient presented seizure + PNES

Table 2. Diagnostic categories before and after vEEG. $(n=125)$

\begin{tabular}{|l|c|c|c|}
\hline Diagnosis & Before VEM & After VEM & Change \\
\hline Epilepsy & $67(54 \%)$ & $51(41 \%)$ & $-16(24 \%)$ \\
\hline Physiological events & $45(36 \%)$ & $50(40 \%)$ & $+5(11.1 \%)$ \\
\hline PNES & $10(8 \%)$ & $18(14.4 \%)$ & $+8(80 \%)$ \\
\hline PNES + Epilepsy & $3(2.4 \%)$ & $5(4 \%)$ & $+2(67 \%)$ \\
\hline
\end{tabular}

Change is defined as After_VEM(variable)-Before_VEM(variable)

Table 3. Demographics and clinical comparation between diagnosis groups.

\begin{tabular}{|l|c|c|c|c|c|c|}
\hline & \multirow{2}{*}{$\begin{array}{c}\text { Epileptic } \\
\text { seizure }\end{array}$} & $\begin{array}{c}\text { Physiological } \\
\text { events } \\
(\mathbf{n}=\mathbf{5 1})^{*}\end{array}$ & $\begin{array}{c}\text { PNES } \\
(\mathbf{n}=\mathbf{5 0})\end{array}$ & & \multicolumn{3}{|c|}{ P value (MW $)$} & ES vs PE & $\begin{array}{c}\text { ES vs } \\
\text { PNES }\end{array}$ & $\begin{array}{c}\text { PE vs } \\
\text { PNES }\end{array}$ \\
\hline Female & $35(70 \%)$ & $29(58 \%)$ & $15(88 \%)$ & 2.000 & $<\mathbf{0 . 0 0 1}$ & $<\mathbf{0 . 0 0 1}$ \\
\hline Age (yrs) & $40.6 \pm 2.6$ & $48 \pm 2.4$ & $36.3 \pm 3.0$ & $0.072^{* *}$ & $0.265^{* *}$ & $\mathbf{0 . 0 1 0}$ \\
\hline Age at onset (yrs) & $26.2 \pm 2.9$ & $42.5 \pm 2.5$ & $30.6 \pm 3.6$ & $<\mathbf{0 . 0 0 1}$ & 0.471 & $\mathbf{0 . 0 2 5}$ \\
\hline Duration (yrs) & $14.4 \pm 2.1$ & $5.8 \pm 1.0$ & $5.9 \pm 2.1$ & $\mathbf{0 . 0 0 8}$ & 0.090 & 0.618 \\
\hline $\begin{array}{l}\text { Abnormal non- } \\
\text { epileptic EEG }\end{array}$ & $5(13 \%)$ & $11(31 \%)$ & $5(35 \%)$ & 2.000 & 1.000 & 2.000 \\
\hline Epileptic EEG & $20(53 \%)$ & $7(20 \%)$ & $4(28 \%)$ & $<\mathbf{0 . 0 0 1}$ & $<\mathbf{0 . 0 0 1}$ & 1.000 \\
\hline Abnormal brain MRI & $31(59.6 \%)$ & $18(41 \%)$ & $7(46.6 \%)$ & $<\mathbf{0 . 0 0 1}$ & $<\mathbf{0 . 0 0 1}$ & $<\mathbf{0 . 0 0 1}$ \\
\hline
\end{tabular}

MW: Mann-Whitney test.

"The patients with diagnosis of Epilepsy and PNES (5) were excluded.

Student-t test. 
to sex proportion, age, age at onset, and abnormal findings in EEG. Paired comparison by groups yielded highly significant differences for ES vs. physiological events $\left(x^{2}=40.65, \mathrm{p}<0.001\right)$, ES vs. PNES $\left(x^{2}=\right.$ $37.30, \mathrm{p}<0.001)$, and physiological events vs. PNES $\left(x^{2}=38.17, \mathrm{p}<\right.$ 0.001 ), with 5 degrees of freedom.

\section{Utility}

We considered that VEM was useful for the clinician when the results of the study led to a change in the previous diagnosis, in treatment or both or when a suspected but not well-defined diagnosis was reinforced by the study.

According to our classification, VEM was defined as useful in 112 patients $(89.6 \%)$. Specifically, treatment was changed $\left(p_{2}\left(T_{2}, d_{1}\right)\right)$ in 32 cases, diagnosis was modified in 10 patients $\left(p_{2}\left(T_{1}, d_{2}\right)\right)$, both of them were modified in 35 patients $\left(p_{2}\left(T_{2}, d_{2}\right)\right)$, and the suspected diagnosis was confirmed in 35 patients $\left(p_{2, c o n}\left(T_{1}, d_{1}\right)\right)$. Therefore, after VEM, the treatment was modified in 67 patients $\left(p_{2}\left(T_{2}, d_{1}\right)+p_{2}\left(T_{2}, d_{2}\right)\right)$ and diagnosis changed in 45 patients $\left(p_{2}\left(T_{1}, d_{2}\right)+p_{2}\left(T_{2}, d_{2}\right)\right)$.

In $10.4 \%$ of cases, the VEM results were inconclusive and could not be termed as useful.

Regarding the group of patients with a final diagnosis of epilepsy $(n=51), 44$ of the patients $(86.3 \%)$ were on at least one AED at the time of admission. After VEM, change in therapy was reported in 33 patients $(64.7 \%)$ of the total group, among whom a new AED was introduced or the dose was increased in 26 patients $(78 \%)$ and the dose was reduced in 7 patients $(21 \%)$.

With respect to the group of patients with physiological events (n $=50), 21$ patients $(42 \%)$ had been on at least one AED at the time of the study. After VEM, the treatment was modified in 18 patients (36\%) with a discontinued or decreased treatment in 15 patients (71\%). There were 12 patients diagnosed with PNES who had previous AED treatment. Discontinuation of therapy was seen in 3 patients.

\section{Discussion}

We have shown that VEM is an extremely helpful tool in the diagnosis and therapeutic management of patients with paroxysmal behavioral events. Misdiagnosis and misclassification of nonepileptic paroxysmal events and ES can lead to inappropriate treatment. High costs have been incurred annually on diagnostic evaluations, inappropriate antiepileptic drugs, and emergency unit utilization [11].

In many cases, clinical information alone can be incomplete or misleading due to descriptions made by untrained witnesses. Furthermore, the correct diagnosis may not be apparent during the short period of outpatient EEG. VEM helps to correlate electroencephalographic changes with clinical events and detect epileptiform activity in long-term records, which also include a sleep period $[2,12]$.

VEM provided a useful yield of recorded clinical events. We have found that the 24-h VEM is able to detect typical clinical events in a $49 \%$ of cases. ES represents $36 \%$ of the cases, which is comparable to other series [13]. Most of the recorded events corresponded to nonepileptic paroxysmal events (62\%), although one-third (21\%) were of psychogenic origin. Thus, an accurate correlation between electroclinical findings is essential to properly characterize different paroxysmal events $[10,14]$. This finding was revealed in a metaanalysis of 135 published studies on VEEG, which describe that 59\% of referrals were for diagnostic reasons [15].

In this sense, PNES has a special importance. VEM is an indispensable tool because it allows simultaneous analysis of both clinical and ictal EEG findings to make the most accurate differential diagnosis between seizure and PNES $[4,16]$. The results of VEM between patients with epilepsy and PNES revealed a sustained decline in AED use from discharge to follow-up [17], suggesting that VEM may contribute to a beneficial elimination of unnecessary medications in the PNES group once a definitive diagnosis is made.

It has been previously shown that in those patients with a change in diagnosis, the most common change involves distinguishing epilepsy from physiological events (68.2\%) [18]. In our work, we found that VEM reduced the previous diagnosis of epilepsy in $24 \%$ of the cases. The reference physicians are more likely to misdiagnose nonepileptic events as seizures than the opposite. This finding may be due to a diagnosis that was made based on clinical history and a routine EEG that can be deceptive. Physiological paroxysmal events were more frequently misdiagnosed as PNES.

A total of $35 \%$ of patients saw their previous diagnosis change. Other authors [19] described a change in the diagnosis in 58\% of patients. The higher figure could possibly be observed because these researchers' studies were longer (1-13 days) and included patients with refractory epilepsy and who were gradually tapering AED. However, recent studies indicate that the VEEG clarifies diagnosis in $56.3 \%$ of patients and changes the diagnosis in $35.6 \%$ of patients [ 15 , 20]. We have shown that VEM was useful in establishing or shaping the diagnosis in 112 patients (89\%). This technique helped to confirm the reference diagnosis with certainty, classify patients with ES, and select the best treatment according to each diagnosis of either epilepsy or nonepileptic events. The high diagnostic yield of VEM in adult patients with recurrent and unprovoked events has been confirmed previously [21-23]; however, the diagnostic usefulness is widely variable $(19 \%-75 \%)$ due to a variation in the definition of utility [10].

We found in the PNES group that women were more frequent in comparison to the other two groups. They were also younger at the time of the study and had a younger age of onset of events compared to those experiencing physiological events. We found a longer disease duration in epilepsy patients, as has been previously described [24], showing a delay in diagnostic confirmation. On the other hand, physiological event patients were older at the onset of symptoms compared to the PNES group, which is probably related to the etiology [25]. It is quite interesting to observe that all three groups are really different. This fact can help to establish a predictive model based on electroclinical findings to help the clinician to classify a patient in daily practice.

We had a final diagnosis of event of physiological origin in $40 \%$ of our patients and of PNES in $14 \%$ of the cases (see table 3 ). It is important 
to highlight that in both these groups, we found epileptiform activity in $20 \%$ and $28 \%$ of cases, respectively, and in both, $\geq 30 \%$ of abnormal nonepileptic activity. There are some studies describing between $17 \%$ and $26 \%$ of patients eventually being diagnosed with nonepileptic events who had interictal epileptiform discharges (IEDs) recorded during VEEG [26, 27]. Similar findings were caused by overreading a standard EEG as abnormal [27-30]. In a study that analyzed the significance of epileptiform abnormalities in patients without epilepsy, the researchers found that of 521 patients with a follow-up of 230 person-years with no history of unprovoked seizures, 64 (12.3\%) had IEDs on their EEG. These patients had associated structural neurological conditions (e.g., tumors and vascular disorders), which would explain the $20 \%$ of epileptiform abnormalities found in our patients with physiological events.

VEM also helped to determine the best treatment for the individual patient based on the type of witnessed events and the electrographic characteristics in VEM. This finding caused a treatment change in $50 \%$ of patients. Moreover, the largest change was seen in the group of patients diagnosed with epilepsy. This finding shows the value of VEM when it comes to influencing the overall care pathway of patients. Optimization of AED may result in avoiding drug adverse events, a better quality of life, and reduction in health costs [31]. In our results, the discontinuation of AED treatment in PNES was low for what we might have expected. The reasons can be different, but it is essential to mention that some clinical physicians often analyze VEM results in an imprecise way for the diagnosis of PNES [32], even though VEM is the gold standard for diagnosis of PNES [4].

Despite being considered an expensive technique with limited availability (a neurophysiologist is needed with healthcare staff and specialized technical equipment) [33], VEM has been demonstrated to be a useful test with robust therapeutic benefits. There are recent recommendations and algorithms based on high-level evidence for the use of VEM for the diagnosis and monitoring of patients with epilepsy[34]. Conversely, the financial and social cost of unclassifiable behavioral disturbances to the patient and the family is considerable, and poorly controlled ES has been associated with impaired psychosocial skills and an increased risk of death [35]. Therefore, in the absence of a study that compares the cost-benefit of VEM and the economic and social cost of chronic uncontrolled seizures (epileptic or nonepileptic), it seems logical to consider that VEM should be a mandatory tool in the differential diagnosis of ES.

\section{Acknowledgement}

This work was financed by a grant from the Ministerio de Sanidad FIS PI17/02193 and was partially supported by FEDER (Fonds Europeen de Developpement Economique et Regional).

\section{References}

1. Tatum WO, Rubboli G, Kaplan PW, Mirsatari SM, Radhakrishnan K et al. (2018) Clinical utility of EEG in diagnosing an monitoring epilepsy in adults. Clin Neurophysiol 129: 1056-1082. [crossref]

2. Fisher RS, Acevedo C, Arzimanoglou A, Bogacz A, Cross JH et al. (2014) ILAE official report: a practical clinical definition of epilepsy. Epilepsia 55: 475-82. [crossref]

3. Krumholz A (1999) Nonepileptic seizures: diagnosis and management. Neurology 53: 76-83. [crossref]
4. Vega-Zelaya L, Alvarez M, Ezquiaga E, et al. (2014) Psychogenic Non-Epileptic Seizures in a Surgical Epilepsy Unit: Experience and a Comprehensive Review. Epilepsy Topics. Prof. Mark Holmes (Ed.) InTech.

5. Cuthill FM, Espie CA (2005) Sensitivity and specificity of procedures for the differential diagnosis of epileptic and non-epileptic seizures: a systematic review. Seizure 14: 293-303. [crossref]

6. Velis D, Plouin P, Gotman J, et al. (2007) Recommendations regarding the requirements and applications for long-term recordings in epilepsy. Epilepsia 48: 379-384. [crossref]

7. Park KI, Lee SK, Chu K, Jung Ju Lee, Dong Wook Kimet al. (2009) The value of video-EEG monitoring to diagnose juvenile myoclonic epilepsy. Seizure 18: 94-99. [crossref]

8. Delgado Nunes V, Sawyer L, Neilson J, Grammati Sarri, J Helen Cross et al. (2012) Diagnosis and management of the epilepsies in adults and children: summary of updated NICE guidelines. $\mathrm{Br} \mathrm{Med} \mathrm{J} \mathrm{344:} \mathrm{281.} \mathrm{[crossref]}$

9. Pastor J, Ortega GJ, Herrera-Peco I, Marta Navas-García, Eduardo G Navarrete et al. (2010) Differential contribution of preoperatory studies to diagnosis in temporal lobe epilepsy surgery. Rev Neurol 51: 393-402. [crossref]

10. Alving J, Beniczky S (2009) Diagnostic usefulness and duration of the inpatient longterm video-EEG monitoring: findings in patients extensively investigated before the monitoring. Seizure 18: 470-473. [crossref]

11. Koblar, SA, Black, AB and Schapel, GJ (1992) Video-audio/EEG monitoring in epilepsy-The Queen Elizabeth Hospital Experience. Clinical and Experimental Neurology 29: 70-73. [crossref]

12. Pastor J (2011) Vídeo-electroencefalografía de scalp. En: Neurocirugía Funcional y Estereotáxica. Eds: E.G. Navarrete y R. G. Sola. Viguera Editores Barcelona; pp: 109-120.

13. McGonigal A, Russell AJC, Mallik AK, M Oto, R Duncanet al. (2004) Use of short term video EEG in the diagnosis of attack disorders. J Neurol Neurosurg Psychiatry 75: 771-772. [crossref]

14. Lawley A, Manfredonia F, Cavanna AE (2016) Video-ambulatory EEG in a secondary care center: A retrospective evaluation of utility in the diagnosis of epileptic and nonepileptic seizures. Epilepsy \& Behavior 57: 137-140. [crossref]

15. Sauro KM, Wiebe N, Macrodimitris S, Wiebe S, Lukmanji S et al. (2016) Quality and safety in adult epilepsy monitoring units: A systematic review and meta-analysis. Epilepsia 57: 1754-1770. [crossref]

16. Gallego I, Ezquiaga E, Betancor D, et al. (2011) Psychogenic non-epileptic seizures in an epilepsy surgery unit. Rev Neurol 52: 449-446. [crossref]

17. Zhang YC, Bromfield EB, Hurwitz S, Aaron Nelson, Kristen Sylvia et al. (2009) Comparison of outcomes of video/EEG monitoring between patients with epileptic seizures and those with psychogenic nonepileptic seizures. Epilepsy Behav 15: 303 307. [crossref]

18. Yogarajah M, Powell HWR, Heaney D, et al. (2009) Medium term monitoring in refractory epilepsy: the Gowers Unit experience. J Neurol Neurosurg Psychiatry 80: 305-311.

19. Ghougassian DF, d'Souza W, Cook MJ, Terence J O'Brien (2004) Evaluating the utility of inpatient video-EEG monitoring. Epilepsia 45: 928-932. [crossref]

20. Sauro KM, Macrodimitris S, Krassman C, et al. (2014) Epilepsy Monitoring Unit Quality Improvement Team. Quality indicators in an epilepsy monitoring unit. Epilepsy Behav 33: 7-11.

21. Alsaadi TM, Thieman C, Shatzel A, Sarah Farias (2004) Video-EEG telemetry can be a crucial tool for neurologists experienced in epilepsy when diagnosing seizure disorders. Seizure 13: 32-34. [crossref]

22. Lee Y, Lee M, Chen I, Yu-Tai Tsai, Chung-Yang Sunget al. (2009) Long-term VideoEEG Monitoring for Paroxysmal Events. Chang Gung Med J 32: 305-312. [crossref]

23. Mohan KK, Markand ON, Salanova V (1996) Diagnostic utility of video EEG monitoring in paroxysmal events. Acta Neurol Scand 94: 320-325. [crossref]

24. Foong M, Seneviratne U (2016) Optimal duration of video-electroencephalographic monitoring to capture seizures. J Clin Neurosci 28: 55-60. [crossref]

25. Mari F, Di Bonaventura C, Vanacore N, Jinane Fattouch, Anna Elisabetta Vaudanoet al. (2006) Video-EEG study of psychogenic nonepileptic seizures: Differential characteristics in patients with and without epilepsy. Epilepsia 5: 64-67. [crossref]

26. McBride AE, Shih TT, Hirsch LJ (2002) Video-EEG monitoring in the elderly: a review of 94 patients. Epilepsia 43: 165-169. [crossref] 
27. Friedman DE, Hirsch LJ (2009) How long does it take to make an accurate diagnosis in an epilepsy monitoring unit? J Clin Neurophysiol 26: 213-217. [crossref]

28. Benbadis SR, Tatum WO (2003) Overintepretation of EEGs and misdiagnosis of epilepsy. J Clin Neurophysiol 20: 42-44. [crossref]

29. Benbadis SR (2013) “Just like EKGs!" Should EEGs undergo a confirmatory interpretation by a clinical neurophysiologist? Neurology $80: 47-51$. [crossref]

30. Benbadis SR (2007) Errors in EEGs and the misdiagnosis of epilepsy: importance, causes, consequences, and proposed remedies. Epilepsy Behav 11:257-2562. [crossref]

31. Manfredonia F, Lawley A, Cavanna AE (2016) Impact of video-ambulatory electroencephalography on the medical management of epilepsy. J Neurol Sci 365 139-1342. [crossref]
32. Harden CL, Burgut FT, Kanner AM (2003) The diagnostic significance of video-EEG monitoring findings on pseudoseizure patients differs between neurologists and psychiatrists. Epilepsia 44: 453-456. [crossref]

33. Parnell KJ, Cascino GD, So EL, et al. (1999) Long-term EEG monitoring in patients with spells: clinical characteristics and predictive factors. Neurology 52: 371-372.

34. Cho YW, Motamedi GK, Kim KT (2019) The clinical utility of non-invasive videoelectroencephalographic monitoring has been diversifying. Neurol Sci 40: 2625-2631. [crossref]

35. Morrel MJ, Pedley TA (2000) “The Scarlet E”: epilepsy is still a burden. Neurology 54: 1882-1883. [crossref]

Jesús Pastor (2020) Utility of Video Electroencephalography Monitoring for Diagnosis of Epilepsy and Nonepileptic Paroxysmal Events.. ARCH Women Health Care Volume 3(3): 1-6. 